\title{
Where to put your best foot forward: Psycho-physiological responses to walking in natural and urban environments
}

*Christopher J Gidlow, ${ }^{1}$ Marc V Jones, ${ }^{1}$ Gemma Hurst,,${ }^{1}$ Daniel Masterson, ${ }^{1}$ David Clark-

Carter, ${ }^{1}$ Mika P Tarvainen, ${ }^{2}$ Graham Smith, ${ }^{1}$ Mark Nieuwenhuijsen ${ }^{3}$

${ }^{1}$ Staffordshire University, Stoke on Trent, United Kingdom

${ }^{2}$ University of Eastern Finland, Kuopio, Finland

${ }^{3}$ Centre for Research in Environmental Epidemiology (CREAL), Barcelona, Spain

\section{CORRESPONDING AUTHOR}

* Christopher J Gidlow, Centre for Sport, Health and Exercise Research, Staffordshire University, Stoke on Trent, United Kingdom, c.gidlow@staffs.ac.uk

\section{KEYWORDS}

Environment; nature; walking; stress; cognitive

Please cite article in press as: Gidlow, C.J., et al., Where to put your best foot forward: Psycho-physiological responses to walking in natural and urban environments. Journal of Environmental Psychology (2015), http:// doi:10.1016/j.jenvp.2015.11.003

Please note: this version is pre-final proofing and withouth publisher's formatting. For final version please see: http://www.sciencedirect.com/science/article/pii/S0272494415300438 


\section{HIGHLIGHTS}

- Non-stressed adults walked in natural (with/without water) and pleasant urban environments

- Mood improved in natural and urban environments

- Salivary cortisol reduced in natural and urban environments

- Restorative experience was higher in natural environments

- Greater cognitive benefits of natural environments were seen 30 min after leaving the environment. 


\section{ABSTRACT}

There is convergent evidence that natural environments allow restoration from stress. This randomised, cross-over field-based trial compared psychological and physiological responses of unstressed individuals to self-paced 30-minute walks in three pleasant environments: residential (urban), natural (green), and natural with water (blue). Changes from baseline (T1) to T2 (end of 30-minute walk), and T3 (30 minutes after leaving environment) were measured in terms of mood, cognitive function, restoration experiences, salivary cortisol, and heart rate variability (HRV). In the final sample ( $\mathrm{n}=38 ; 65 \%$ male; mean age $40.9 \pm 17.6$ years), mood and cortisol improved at T2 and T3 in all environments. Green and blue environments were associated with greater restoration experiences, and cognitive function improvements that persisted at T3. Stress reduction (mood and cortisol changes) in all environments points to the salutogenic effect of walking, but natural environments conferred additional cognitive benefits lasting at least 30 minutes after leaving the environment. 


\section{Introduction}

There is a convergent evidence for a positive relationship between natural environment exposure and health (Hartig, Mitchell, de Vries, \& Frumkin, 2014). Commonly posited explanatory mechanisms include increased opportunities for physical activity and social interaction from active use of natural environments, stress reduction and cognitive restoration of passive or active use, and mitigation of environmental pollutants, such as noise and air pollution (Bowler, Buyung-Ali, Knight, \& Pullin, 2010; Hartig et al., 2014; Nieuwenhuijsen et al., 2014).

Experimental studies to characterise the benefits of engaging with natural environments have typically departed from one of two theoretical standpoints: attention restoration theory (ART), which proposes that nature allows restoration from directed attention fatigue and enable more effective cognitive performance (Kaplan, 1995); stress reduction theory (SRT), where natural environments are thought to influence affective states by promoting recovery from stress, and diminishing arousal and negative thoughts through psycho-physiological pathways (Ulrich et al., 1991; Ulrich, 1983).

ART is well supported by data from laboratory-based image viewing studies (Berto, 2005; Staats, Kieviet, \& Hartig, 2003; van den Berg, Koole, \& van der Wulp, 2003) and some field studies (Berman, Jonides, \& Kaplan, 2008; Hartig, Evans, Jamner, Davis, \& Gärling, 2003; Sonntag-Öström et al., 2014; Tennessen \& Cimprich, 1995) showing that attention, measured as performance at cognitive tasks, is better when individuals are exposed to natural rather than urban environments. For SRT, evidence to date suggests that viewing or visiting natural environments can result in better affective outcomes measured through self-reported mood scales (Bowler et al., 2010; Lee et al., 2011; Tsunetsugu et al., 2013; Tyrväinen et al., 2014), perhaps more so when the natural environment contains water, such as a river, lake or coast (Barton \& Pretty, 2010). 
There is growing interest in measuring corresponding physiological responses to natural environments, particularly in field (rather than laboratory) studies, where the experiences of participants in the environments can be more ecologically valid and typically involve walking (sometimes in addition to sitting) in urban and natural environments. To date, the evidence remains less conclusive (Bowler et al., 2010). Physiological measures in field experiments have been used to characterise cardiovascular (e.g., heart rate, blood pressure, heart rate variability) and neuroendocrinal (e.g., cortisol, salivary amylase) responses, and more recently, brain activity (Aspinall, Mavros, Coyne, \& Roe, 2015). There is evidence that walking in natural environments can lower blood pressure (Hartig et al., 2003), but evidence for stress lowering effects of walking in natural environments measured through reduced cortisol concentrations, is inconsistent (Beil \& Hanes, 2013; Bowler et al., 2010; Lee et al., 2011; Park, Tsunetsugu, Kasetani, Kagawa, \& Miyazaki, 2010). Similarly, despite support from laboratory studies involving walking in simulated environments (Annerstedt et al., 2013) and from seated image viewing studies (Brown, Barton, \& Gladwell, 2013; Gladwell et al., 2012), there is not strong support for beneficial heart hate variability (HRV) responses to natural versus urban environments from field experiments (Bowler et al., 2010). Overall, our understanding of the cardiovascular and neuroendocrinal responses to natural environments in field studies remains limited.

We present data from a field-based, randomised, cross-over experiment that addresses the call for robust experimental examination of psycho-physiological responses to natural environments (Bowler et al., 2010) and contributes to the emerging literature in a number of ways. First, it is not uncommon for field studies in this area to use comparator urban environments that are inherently stressful. We avoided using busy commercial areas and main roads with high traffic volumes for our urban comparator, to reduce the risk of detecting negative responses to the urban condition, rather than positive responses to natural 
environments (Hartig et al., 2003; Lee et al., 2011; Park et al., 2010; Tsunetsugu et al., 2013; Tyrväinen et al., 2014). Second, we explored instorative effects of natural environments, which have been described as the benefits that 'do not necessarily follow a reduction in adaptive capacities nor involve restoring diminished capacities' (Hartig, 2007, p.2). Rather than focusing on restoration from a stressed or depleted baseline state, our interest was the potential for natural environments to promote psycho-physiological improvements in a non-stressed, healthy adult sample, as engagement with natural environments may have wider public health benefits than facilitating recovery from stress or directed attention fatigue. Third, we controlled for potential confounding effects of social interaction and physical activity. Finally, our experimental design enabled enquiry beyond the immediate exposure effects to understand whether any immediate benefits were sustained once people left the environment.

The overarching study aims were to: (i) to compare psycho-physiological responses to natural environments with and without water, and a pleasant urban environment; (ii) explore the immediate and sustained instorative potential of natural environments. In the context of existing research, it was hypothesised that natural environments would confer more favourable responses that urban, with strongest effects in natural environments with water present. However, given our focus on healthy, unstressed individuals, and a pleasant urban comparator environment, such effects might be less pronounced that reported elsewhere.

\section{Method}

\subsection{Participants}

Participants were adults who lived, worked or studied in a medium size conurbation in the West Midlands region of the UK. Inclusion criteria were: aged $\geq 18$ years; non-smokers; not pregnant; no chronic medical conditions; not taking medication that will influence cortisol 
or heart rate variability (Granger, Hibel, Fortunato, \& Kapelewski, 2009); 'healthy' based on self-report (SF12v2) (Quality Metric, 2006); and able to walk for 30 minutes.

\subsection{Design and procedure}

Data were collected between June and October 2013. We used a randomised, crossover, within-subjects design, whereby all participants walked for 30 minutes in each of three environments (Figure 1) at the same time on three days. Data were always collected on weekdays (Mon-Fri) and, where possible, all three days completed within two weeks (depending on participant availability). The order of environmental exposures was individually randomised by ascribing a number of 1 to 6 to represent each possible environment combination, which was then assigned to the participant using a random number generator (MS Excel). Although participants were not given prior warning of the order in which environments would be visited, blinding of allocation was not relevant given the nature of the trial (i.e., exposures involved visiting an environment; all participants visited all environments). Environment visits were only conducted in temperate conditions and were re-arranged in the event of rain/inclement weather conditions. Two researchers $(\mathrm{GH}, \mathrm{DM})$ controlled these procedures, with the same researcher being assigned to participants for all of their environmental visits.

Participants were recruited through local media, advertising in and around the University campus, and a mail shot to households within $1 \mathrm{~km}$ of the campus, with eligibility screening via an online survey. This approach was intended to improve generalisability, rather than delimiting to a student sample. Eligible participants were invited to attend the University at either 12:00 or 14:00 on each day, and to refrain from consuming caffeine or food for at least 60 minutes prior to arrival. Figure 2 illustrates the procedures on each of the three days of data collection. Briefly, following baseline measures (T1), participants were transported to the 
environment to walk along a pre-designated route, at a self-directed pace. Self-pacing was used to optimise participant experience and ecological validity, and walking helped to ensure that exercise intensity was of mostly light intensity (approaching moderate intensity for some). A researcher (GH or DM) accompanied the participant on the walk, remaining half a stride behind to allow the participant to determine the pace. Participants were asked for their Rate of Perceived Exertion (RPE) at five-minute intervals, with no other social interaction. After 30 minutes, follow-up measures were taken (T2), and repeated a further 30 minutes later after returning to the University (T3). Participants were offered a $£ 40$ retail voucher in appreciation of their time. All study procedures were approved by the University Ethics Committee.

\subsection{Environments}

The three environmental conditions were: urban - quiet residential streets with low levels of traffic; green - country park within the city; blue - footpath besides a canal with a range of natural vegetation (Figure 1). Environments were selected on the basis of several criteria: less than 15-minute drive from the University; rated as 'natural' or 'urban' (depending on condition) and 'pleasant' based on responses to a separate online image survey (Gidlow, Jones, Hurst, \& Masterson, 2013); comparable gradient to minimise differences in exercise intensity; and unlikely to have large numbers of people to reduce the risk of incidental social interaction. The roads in the urban environment were residential, had traffic levels that were too low for official measurement and classification in the UK (i.e., $<500$ cars/day), and noise levels comparable to the natural environments (urban 50.56 $\pm 4.25 \mathrm{dbA}$, green $47.47 \pm 2.94 \mathrm{dbA}$, blue $45.60 \pm 1.46 \mathrm{dbA}$, average of two 15 -minute recordings at representative points in each environment measured by a Precision Gold N05CC Sound level meter). The low levels of noise in all environments provided confidence the slightly higher readings in the blue and urban 
environments should not have been sufficient to promote a stress response in the urban environment.

\subsection{Measures}

Baseline profiling. Participants completed a series of questionnaires to profile: sociodemographics (age, gender, ethnicity, education, employment status, postcode); self-reported health using the Short-Form 12 (SF12v2 used to determine Physical and Mental Component Scores, where scores below/above 50 indicate health that is below/above average, respectively);(Quality Metric, 2006) Perceived Stress Scale (PSS) (Cohen \& Williamson, 1988); nature-relatedness using the NR-6 Nature-relatedness scale (Nisbet, Zelenski, \& Murphy, 2009). The latter was included to explore whether the extent to which individuals felt connected with the natural world could explain differential responses to natural environments.

\section{Responses to the Natural Environment.}

- Total Mood Disturbance (TMD) was assessed using the BRUMS (Terry, Lane, \& Fogarty, 2003), a widely used and validated abbreviated version of the Profile of Moods States (McNair, Lorr, \& Droppleman, 1971), where lower values indicate better mood.

- Cognitive function was assessed through the Backward Digit Span (BDS), a measure of working memory used by others in this area (Berman et al., 2008). Participants were read a sequence of three to nine digits and asked to repeat them in reverse order. This was repeated up to 14 times (two repetitions of each digit span) and stopped after two consecutive failures, with the length of the longest correct sequence providing a measure of cognitive function (i.e., higher scores indicate better cognitive function).

- Restoration experience was measured through an abbreviated six-item version of the Restoration Outcome Scale (Korpela, Ylén, Tyrväinen, \& Silvennoinen, 2008), where higher 
scores indicate a more restorative experience. The scale included items, such as 'I feel calmer', 'After visiting this place I feel restored and relaxed', and 'My concentration and alertness clearly increased', to provide a measure of the perceived restorative experience of visiting each environment.

- Salivary cortisol was measured as a physiological marker of stress. Cortisol concentrations were determined from saliva samples collected using synthetic swabs, which participants placed beneath their tongue for two minutes. All samples were centrifuged at 3000rpm for 15 minutes, divided into two samples and stored separately at $-80^{\circ} \mathrm{C}$ until batch analysis (Salimetrics Ltd. High Sensitivity Salivary Cortisol Enzyme Immunoassay Kit). Salivary cortisol concentrations (nmol/l) provided an objective measure of stress, with higher values indicating higher stress.

- Ambulatory Heart Rate (HR) and HRV data were collected using the eMotion monitor (http://www.megaemg.com/products/emotion-hrv) (Heikkinen, 2012). HR was included as an objective measure of exercise intensity. HRV reflects the interplay between the excitatory sympathetic nervous system, which is dominant at times of stress and the inhibitory parasympathetic nervous system, which is dominant in periods of relative safety and restoration (Appelhans and Luecken, 2006). Data processing in Kubios software (http://kubios.uef.fi) included: artefact correction (Tarvainen, Niskanen, Lipponen, Ranta-Aho, \& Karjalainen, 2014) removal of very low frequency trend components using a smoothness priors detrending method (Tarvainen, Ranta-Aho, \& Karjalainen, 2002), and interpolation of the RR series. HRV indicators used in analysis included mean RR interval (time between each heart beat), standard deviation of normal-to-normal intervals (SDNN), and low frequency (LF, 0.04-0.15 Hz) and high frequency (HF, 0.15-0.4 Hz) powers from Fourier spectra. For each HRV variable, the percentage coefficient of component variance $(\% \mathrm{CCV})$ was calculated to obtain $\% \mathrm{CCV}$ to 
control for differences in RR interval as a result of different exercise intensities (Højgaard, Holstein-Rathlou, Agner, \& Kanters, 1998).

- Rate of Perceived Exertion (RPE) was measured at five-minute intervals during the walks using the Borg Scale (Borg, 1990), to provide a measure of participant-rated exercise intensity.

\subsection{Statistical analysis}

For each of the outcome measures, with the exception of restoration (measured T2 only), a three-by-three repeated measures ANOVA was used to investigate the effects of time (baseline, immediately post and 30 minutes after leaving the environment), environment (urban, green, blue) and time by environment interactions. If there was a significant interaction, this was explored using simple main effects via one-way ANOVA comparing environments, and where environment was significant, this was explored using paired contrasts, via t-tests with Tukey's HSD used to control for multiple testing. Where there was a main effect of environment, but no interaction with time, this was again explored via paired contrasts. Physiological measurements required transformation to meet parametric analysis assumptions (cortisol concentration was square-root transformed and HRV parameters were logtransformed). Where assumptions of sphericity were violated, adjusted degrees of freedom using the Greenhouse-Geisser estimate were reviewed. The target sample size was 40 . This was based on: an estimated 20-50 participants required to detect a medium-large effects, using a within subjects ANOVA with three conditions $(\mathrm{df}=2)$, power of .8 and an alpha level of .05 ; the practical considerations of collecting all data in temperate conditions. Analyses were undertaken in IBM SPSS version 22. 


\section{Results}

\subsection{Participants}

Eighty-eight screening questionnaires were completed. Seventeen individuals were excluded as ineligible (unable to attend appointments $n=5$; on medication $n=6$; long-term illness $n=2$; medication and long-term illness $n=3$; smoker $n=1$ ) and 31 declined to take part or did not respond to further communication. Of the 40 participants who started the study, two were lost to follow-up and did not visit all environment; 38 completed and were included in analysis (23 male, 15 female; $M_{\text {age }}=40.9 \pm 17.6$ ). Participants were predominantly White British (92.1\%) and the majority were in full-time work (29\%), students (29\%) or retired individuals (24\%); the remainder comprised unemployed, home-keepers and those in part-time work. Relatively good health of the sample was confirmed in terms of self-reported health (Physical Component score=55.5 \pm 5.08 ; mean Mental Component Score=52.3 \pm 7.8 ), Body Mass Index $\left(25.4 \pm 5.0 \mathrm{kgm}^{-2}\right)$ and mean PSS score (11.1 \pm 6.5$)$.

\subsection{Walking exposure}

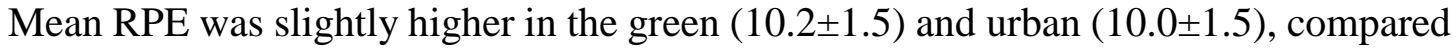
with the blue environment $(9.0 \pm 1.5)$, but all were in the very light to light intensity range. Heart rate data confirmed that participations were walking at moderate intensity; i.e., $55-69 \%$ of maximal HR (Pollock et al., 1998). As a percentage of theoretical maximal heart rate $\left(\mathrm{HR}_{\max }=220\right.$-age), mean $\% \mathrm{HR}_{\max }$ was comparable in each environment (urban $59.97 \pm 8.86 \%$; green $62.18 \pm 9.56 \%$; blue $58.67 \pm 9.35 \%$ ).

\subsection{Psychological responses to environments}

Mood improved from baseline (T1) in all environments, with a significant main effect of time on TMD, $\mathrm{F}(1.37,46.41)=6.40, \mathrm{p}=.009, \eta^{2}=.07$, post-walk (T1 to $\left.\mathrm{T} 2\right), \mathrm{F}(1,34)=8.77$, 
$\mathrm{p}=.006, \eta^{2}=.23$, and 30-mintues after leaving the environment ( $\mathrm{T} 1$ to $\mathrm{T} 3$ ), $\mathrm{F}(1,34)=5.29$, $\mathrm{p}=.028, \eta^{2}=.16$. There was no significant main effect for environment, $\mathrm{F}(2,68)=1.77 \mathrm{p}=.178$, $\eta^{2}=.02$ and no significant environment*time interaction effect, $\mathrm{F}(2.91,98.89)=1.01, \mathrm{p}=.389$, $\eta^{2}=.005($ Table 1$)$.

There was also a significant main effect of time on cognitive function, $F(2,74)=3.39$, $\mathrm{p}=.039, \eta^{2}=.01$, but no significant main effect for environment $\mathrm{F}(2,74)=.926, \mathrm{p}=.401, \eta^{2}=.01$. Additionally, a significant environment*time interaction effect was evident, $F(4,148)=2.89$, $\mathrm{p}=.024, \eta^{2}=.02$, such that improvements in cognitive task performance persisted at T3 following exposure to both natural environments, but reduced to below baseline levels in the urban condition: Blue vs. Urban (T1 vs. T3), $\mathrm{F}(1,37)=9.26, \mathrm{p}=.004$; Green vs. Urban (T2 vs. T3), $\mathrm{F}(1,37)=4.35, \mathrm{p}=.044$ (Table 1, Figure 3).

A significant difference in restoration experiences was also found between environments following the 30-minute walk, $F(2,72)=28.54, p<.001, \eta^{2}=.21$ (Figure 4). Follow-up contrasts showed that ratings of restoration experiences were significantly higher in the natural compared with urban environments: Green vs. Urban, $\mathrm{t}(36)=5.87, \mathrm{p}<.001, \eta^{2}=.49$; Blue vs. Urban, $\mathrm{t}(36)=-6.43, \mathrm{p}<.001, \eta^{2}=.57$.

The influence of participants' trait nature-relatedness was explored for outcomes where a significant environment effect was observed. Nature-relatedness was not significantly correlated with restorative experience in the green or blue environments (all p>.069), or with changes in cognitive function (all $\mathrm{p}>.224$ ). Collectively, the results suggested no potential relationship linking nature-relatedness with restorative experience or cognitive function, so further analyses were not warranted. 
3.4 Physiological responses to environments

A significant main effect of time on cortisol concentration was found, $F(1.37$, 48.01) $=82.83, \mathrm{p}<.001, \eta^{2}=.29$. Significant reductions were observed in all environments at $\mathrm{T} 2$, $\mathrm{F}(1,35)=83.80, \mathrm{p}<.001, \eta^{2}=.71$, and $\mathrm{T} 3, \mathrm{~F}(1,35)=98.23, \mathrm{p}<.001, \eta^{2}=.73$ (Table 1). There was no significant main effect for environment, $\mathrm{F}(2,70)=.195, \mathrm{p}=.823, \eta^{2}=.002$ and no significant environment*time interaction effect, $\mathrm{F}(2.52,88.30)=.801, \mathrm{p}=.478, \eta^{2}=.004$. Tests were run for males and females separately, but there was no evidence of differential cortisol response by gender (data not shown).

Heart rate variability data were inconclusive, showing no consistent patterns by time or between environments for any of the HRV indicators from baseline to T3 (Table 1). Differences in respiration rate were explored through $\mathrm{HF}$ Peak Power $(\mathrm{Hz})$ to determine possible confounding effects, but environment*time effects were not evident (all p>.194).

\section{Discussion}

Our data suggest that walking in a natural environment confers greater benefit for restorative experience and cognitive function, which persisted for at least 30 minutes after leaving the environment, when compared with a similar walk in a pleasant urban environment. However, no differences in self-reported mood, or physiological indicators of stress (cortisol and HRV), were observed.

Perceptions of greater restoration following natural environment visits are consistent with the literature (Beil \& Hanes, 2013; Bodin \& Hartig, 2003; Korpela, Ylén, Tyrväinen, \& Silvennoinen, 2010). Significant environmental differences in cognitive function did not manifest until 30-minutes later when participants had left the environment. In relation to the ART, researchers have shown changes in performance on cognitive tasks do not consistently emerge within 15-20 minutes of natural environment exposure (Laumann, Gärling, \& 
Stormark, 2003), but are manifest after 50 minutes of engagement (Berman et al., 2008; Hartig et al., 2003). In our study, these changes were observed 60 minutes after first engaging with the natural environment (30 minutes after leaving). This has practical implications; for example, a lunchtime walk in a natural environment could help to improve cognitive performance at work for at least 30 minutes after returning.

We did not find environmental differences in indicators of stress assessed by selfreported mood and cortisol. The lack of mood effect consistent with some studies comparing responses to walking in natural and urban environments (Johansson, Hartig, \& Staats, 2011; Kinnafick \& Thøgersen-Ntoumani, 2014). It also supports data showing mood benefits of walking at a self-directed pace (Ekkekakis et al. 2011). Where field-based studies have reported greater improvements in mood following exposure to natural, compared with urban environments, these are potentially attributable to a negative response to the urban condition (Hartig et al., 2003; Lee et al., 2011; Tsunetsugu et al., 2013). Reductions in cortisol were also observed in all environmental conditions, which is consistent with the lack of environmental effects found in the published literature (Beil \& Hanes, 2013; Lee et al., 2011; Tyrväinen et al., 2014). Where beneficial cortisol responses have been reported, there are recognised limitations, such as absence of baseline data (e.g., Park et al., 2010), or other potential confounders, such as differences in activity levels across environments (e.g., Van den Berg \& Custers, 2010). The magnitude of reductions in cortisol we observed over 30 minutes were greater than would be expected from the typical diurnal pattern (Doane, Chen, Sladek, Van Lenten, \& Granger, 2015; Edwards, Clow, Evans, \& Hucklebridge, 2001), which suggests that there were instorative effects although this remains speculative in the absence of an inactive control condition. In any case, as improvements were observed across all conditions, our cortisol data did not support the natural versus urban benefits reported elsewhere. 
Heart rate variability data did not show any differences in environmental response, or any consistent patterns over time. Significant natural-urban environment differences in HRV have been reported in laboratory settings using images and 3-D simulations (Annerstedt et al., 2013; Brown et al., 2013; Gladwell et al., 2012). Field-based research has suggested favourable responses to walking and sitting in forest versus busy urban environments (higher HF; lower LF:HF). However, in the absence of baseline data, caution is required (Park et al., 2010; Tsunetsugu et al., 2013), and where change from baseline has been reported, a negative response to the busy urban environment, rather than positive natural response, is evident (Lee et al., 2011). Based on our data, 30-minutes of walking or low intensity physical activity in natural or pleasant urban environments does not significantly alter HRV. However, as noted below, the amount of noise in HRV when taking measurements in the field, rather than in laboratory conditions, might negate our ability to detect subtle environmental responses using HRV.

In our sample of non-stressed, healthy adults who visited natural and pleasant urban environments, our findings are better explained by ART than SRT. Specifically, the environmental differences that emerged were for restorative experience and cognitive function, the latter emerging 60-minutes post-initial exposure. That we observed stress-reducing effects in all environments points to the salutogenic effects of being physically active, even at low intensity. The improvements to mood across all environments and of cognitive function in the natural environments may reflect the motivational properties of physical activity (Ekkekakis, 2003). Also, the physical activity might have such a dominant influence that more subtle environmental effects were not detected. This potential limitation is particularly relevant to HRV. Although we used the \%CCV indices to account for differences in heart rate (i.e., exercise intensity) and explored differences in respiration rate, additional controls were not feasible. Further we recognise that it was not possible to adhere to recommendations for HRV 
measurement. Efforts to make the participant experience as normal as possible and to avoid boredom, meant that we were unable to measure HRV during timed breathing for five-minute periods, and used one-minute segments of untimed breathing as used elsewhere (Lee et al., 2011).

Other limitations are acknowledged. First, it was not feasible to explore the full range of different environments or to recruit a fully representative sample, which limits generalisability. Rather, we recruited a range of heathy, non-stressed adults and selected environments with specific attributes (as detailed earlier). Second, while environment visits were only conducted in temperate conditions, some variability in weather between days could have influenced participant experiences. Third, it would have been preferable, but not feasible to standardise exercise intensity across all participants and environments. As detailed earlier, self-paced walking was chosen to optimise participant experience and ecological validity, whilst helping to ensure that exercise mostly light intensity (so avoiding problems of psychophysiological impacts of higher intensity activity). Fourth, the researcher walking with the participant was necessary, but could have made the experience feel somewhat unusual. Measures to limit this included having the same researcher-participant pairing throughout and randomising the order of exposures (to negate issues of the experience feeling less unusual on subsequent days).

Our study was concerned with the potential instorative effects in individuals who are not stressed or cognitively fatigued (Hartig, 2007), and used a comparator urban environment that was not stressful or unpleasant. This comes from a public health perspective, that engagement with natural environments may have wider public health benefits than facilitating recovery from stress. The applicability of research in this area could be enhanced by replicating how people typically engage with natural environments. In particular, people tend to visit the same natural environments repeatedly so understanding whether the benefits of a single 
exposure are attenuated, maintained or increased is an area of future focus. Further research could include a broader range of urban and natural environments to begin mapping environmental characteristics to stress-reducing capacity. For example, a recent study has shown that the relationship between tree coverage and stress recovery was non-linear, such that stress recovery improved with increasing tree coverage up to a point, but decreased thereafter (Jiang et al. 2014). Exploring the longevity of the positive effects on cognitive function from being physically active in a pleasant natural environment would be another area for future research, specifically to determine if these extend beyond the 60 minutes post-engagement shown in the present study.

Overall, our findings indicate that light intensity physical activity in a natural environment confers greater benefit for restorative experience and cognitive function, which persisted for at least 30 minutes after leaving the environment, when compared with a similar walk in an urban environment. For immediate improvements in measures of well-being you can put your best foot forward in a pleasant urban or natural environment, but for the additional improvement in cognitive function, choose a natural environment. 


\section{References}

Annerstedt, M., Jönsson, P., Wallergård, M., Johansson, G., Karlson, B., Grahn, P., . . . Währborg, P. (2013). Inducing physiological stress recovery with sounds of nature in a virtual reality forest - results from a pilot study. Physiology \& Behavior, 118, 240-250.

Appelhans, B. M., \& Luecken, L. J. (2006). Heart rate variability as an index of regulated emotional responding. Review of General Psychology, 10, 229.

Aspinall, P., Mavros, P., Coyne, R., \& Roe, J. (2015). The urban brain: Analysing outdoor physical activity with mobile EEG. British Journal of Sports Medicine, 49, 272-276.

Barton, J., \& Pretty, J. (2010). What is the best dose of nature and green exercise for improving mental health? A multi-study analysis. Environmental Science \& Technology, 44, 3947-3955.

Beil, K., \& Hanes, D. (2013). The influence of urban natural and built environments on physiological and psychological measures of stress - A pilot study. International Journal of Environmental Research and Public Health, 10, 1250-1267.

Berman, M., Jonides, J., \& Kaplan, S. (2008). The cognitive benefits of interacting with nature. Psychological Science, 19, 1207-1212.

Berto, R. (2005). Exposure to restorative environments helps restore attentional capacity. Journal of Environmental Psychology, 25, 249-259.

Bodin, M., \& Hartig, T. (2003). Does the outdoor environment matter for psychological restoration gained through running? Psychology of Sport and Exercise, 4, 141-153.

Borg, G. (1990). Psychophysical scaling with applications in physical work and the perception of exertion. Scandinavian Journal of Work, Environment and Health, 16(Suppl 1), 55-58. 
Bowler, D., Buyung-Ali, L., Knight, T., \& Pullin, A. (2010). A systematic review of evidence for the added benefits to health of exposure to natural environments. BMC Public Health, $10,456$.

Brown, D. K., Barton, J. L., \& Gladwell, V. F. (2013). Viewing nature scenes positively affects recovery of autonomic function following acute-mental stress. Environmental Science \& Technology, 47, 5562-5569.

Cohen, S., \& Williamson, G. (1988). Perceived stress in a probability sample of the United States. In S. Spacapan, \& S. Oskamp (Eds.). The Social Psychology of Health. The Claremont Symposium on Applied Social Psychology (pp. 31-67). Thousand Oaks, CA, US: Sage Publications.

Doane, L. D., Chen, F. R., Sladek, M. R., Van Lenten, S. A., \& Granger, D. A. (2015). Latent trait cortisol (LTC) levels: Reliability, validity, and stability. Psychoneuroendocrinology, $55,21-35$.

Edwards, S., Clow, A., Evans, P., \& Hucklebridge, F. (2001). Exploration of the awakening cortisol response in relation to diurnal cortisol secretory activity. Life Sciences, 68, 20932103.

Ekkekakis, P. (2003). Pleasure and displeasure from the body: Perspectives from exercise. Cognition \& Emotion, 17, 213-239.

Ekkekakis, P., Parfitt, G., \& Petruzzello, S. J. (2011). The pleasure and displeasure people feel when they exercise at different intensities. Sports Medicine, 41, 641-671.

Gidlow, C. J., Jones, M. V., Hurst, G. L., \& Masterson, D. Individual responses to natural and urban environments. Conference of the International Society of Environmental Epidemiology (ISEE) 2013. Basel, Switzerland, 
Gladwell, V. F., Brown, D. K., Barton, J. L., Tarvainen, M. P., Kuoppa, P., Pretty, J., . . . Sandercock, G. R. H. (2012). The effects of views of nature on autonomic control. European Journal of Applied Physiology, 112, 3379-3386.

Granger, D. A., Hibel, L. C., Fortunato, C. K., \& Kapelewski, C. H. (2009). Medication effects on salivary cortisol: Tactics and strategy to minimize impact in behavioral and developmental science. Psychoneuroendocrinology, 34, 1437-1448.

Hartig, T. (2007). Three steps to understanding restorative environments as health resources. Open Space: People Space, 163-179.

Hartig, T., Evans, G. W., Jamner, L. D., Davis, D. S., \& Gärling, T. (2003). Tracking restoration in natural and urban field settings. Journal of Environmental Psychology, 23, 109-123.

Hartig, T., Mitchell, R., de Vries, S., \& Frumkin, H. (2014). Nature and health. Annual Review of Public Health, 35, 207-228.

Heikkinen, O. (2012). Development and validation of an ambulatory HRV measurement system (MSc).

Højgaard, M. V., Holstein-Rathlou, N., Agner, E., \& Kanters, J. K. (1998). Dynamics of spectral components of heart rate variability during changes in autonomic balance. American Journal of Physiology - Heart and Circulatory Physiology, 275, H213-H219.

Jiang, B., Chang, C. Y., \& Sullivan, W. C. (2014). A dose of nature: Tree cover, stress reduction, and gender differences. Landscape and Urban Planning, 132, 26-36.

Johansson, M., Hartig, T., \& Staats, H. (2011). Psychological benefits of walking: Moderation by company and outdoor environment. Applied Psychology: Health and Well-being, 3, 261-280.

Kaplan, S. (1995). The restorative benefits of nature: Toward an integrative framework. Journal of Environmental Psychology, 15, 169-182. 
Kinnafick, F., \& Thøgersen-Ntoumani, C. (2014). The effect of the physical environment and levels of activity on affective states. Journal of Environmental Psychology, 38, 241-251.

Korpela, K. M., Ylén, M., Tyrväinen, L., \& Silvennoinen, H. (2008). Determinants of restorative experiences in everyday favorite places. Health \& Place, 14, 636-652.

Korpela, K. M., Ylén, M., Tyrväinen, L., \& Silvennoinen, H. (2010). Favorite green, waterside and urban environments, restorative experiences and perceived health in Finland. Health Promotion International, 25, 200-209.

Laumann, K., Gärling, T., \& Stormark, K. (2003). Selective attention and heart rate responses to natural and urban environments. Journal of Environmental Psychology, 23, 125-134.

Lee, J., Park, B. J., Tsunetsugu, Y., Ohira, T., Kagawa, T., \& Miyazaki, Y. (2011). Effect of forest bathing on physiological and psychological responses in young Japanese male subjects. Public Health, 125, 93-100.

McNair, D. M., Lorr, M., \& Droppleman, L. F. (1971). Manual for the profile of mood states. San Diego: Educational and Industrial Testing Services.

Nieuwenhuijsen, M. J., Kruize, H., Gidlow, C., Andrusaityte, S., Antó, J. M., Basagaña, X., . . Grazuleviciene, R. (2014). Positive health effects of the natural outdoor environment in typical populations in different regions in Europe (PHENOTYPE): A study programme protocol. BMJ Open, 4(4).

Nisbet, E. K., Zelenski, J. M., \& Murphy, S. A. (2009). The nature relatedness scale. Environment and Behavior, 41, 715-740.

Park, B. J., Tsunetsugu, Y., Kasetani, T., Kagawa, T., \& Miyazaki, Y. (2010). The physiological effects of shinrin-yoku (taking in the forest atmosphere or forest bathing): Evidence from field experiments in 24 forests across japan. Environmental Health and Preventive Medicine, 15, 18-26. 
Pollock, M., Gaesser, G., Butcher, J., Després, J., Dishman, R., Franklin, B., \& Garber, C. E. (1998). American college of sports medicine position stand. The recommended quantity and quality of exercise for developing and maintaining cardiorespiratory and muscular fitness, and flexibility in healthy adults. Medicine and Science in Sports and Exercise, 30, 975-991.

Quality Metric. (2006). The SF-12®: An even shorter health survey. Available at: http://www.sf-36.org/tools/sf12.shtml.

Sonntag-Öström, E., Nordin, M.,- Lundell, Y., Dolling, A., Wiklund, U., Karlsson, M., . . . Slunga Järvholm, L. (2014). Restorative effects of visits to urban and forest environments in patients with exhaustion disorder. Urban Forestry \& Urban Greening, $13,344-354$.

Staats, H., Kieviet, A., \& Hartig, T. (2003). Where to recover from attentional fatigue: An expectancy-value analysis of environmental preference. Journal of Environmental Psychology, 23, 147-157.

Tarvainen, M. P., Niskanen, J., Lipponen, J. A., Ranta-Aho, P. O., \& Karjalainen, P. A. (2014). Kubios HRV-heart rate variability analysis software. Computer Methods and Programs in Biomedicine, 113, 210-220.

Tarvainen, M. P., Ranta-Aho, P. O., \& Karjalainen, P. A. (2002). An advanced detrending method with application to HRV analysis. IEEE Transactions on Bio-Medical Engineering, 49, 172-175.

Tennessen, C. M., \& Cimprich, B. (1995). Views to nature: Effects on attention. Journal of Environmental Psychology, 15, 77-85.

Terry, P. C., Lane, A. M., \& Fogarty, G. J. (2003). Construct validity of the profile of mood states-adolescents for use with adults. Psychology of Sport and Exercise, 4, 125-139. 
Tsunetsugu, Y., Lee, J., Park, B., Tyrväinen, L., Kagawa, T., \& Miyazaki, Y. (2013).

Physiological and psychological effects of viewing urban forest landscapes assessed by multiple measurements. Landscape and Urban Planning, 113, 90-93.

Tyrväinen, L., Ojala, A., Korpela, K., Lanki, T., Tsunetsugu, Y., \& Kagawa, T. (2014). The influence of urban green environments on stress relief measures: A field experiment. Journal of Environmental Psychology, 38, 1-9.

Ulrich, R. S., Simons, R. F., Losito, B. D., Fiorito, E., Miles, M. A., \& Zelson, M. (1991). Stress recovery during exposure to natural and urban environments. Journal of Environmental Psychology, 11, 201-230.

Ulrich, R. (1983). Aesthetic and affective response to natural environment. Behaviour and the Natural Environment (pp. 85-125). US: Springer.

Van den Berg, A. E., \& Custers, M. H. G. (2010). Gardening promotes neuroendocrine and affective restoration from stress. Journal of Health Psychology, June 3.

van den Berg, A. E., Koole, S. L., \& van der Wulp, N. Y. (2003). Environmental preference and restoration: (How) are they related? Journal of Environmental Psychology, 23, $135-$ 146. 
4 FIGURES

5

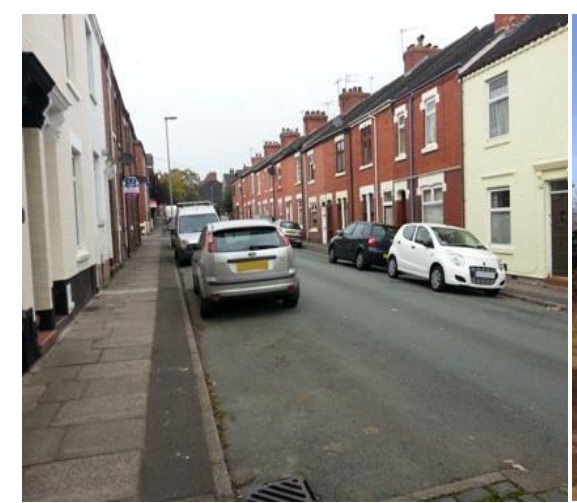

Urban

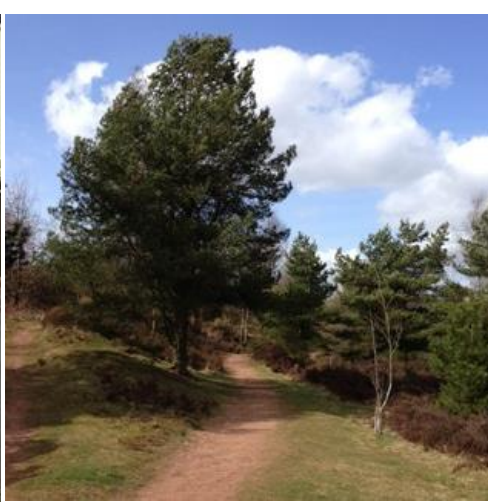

Green

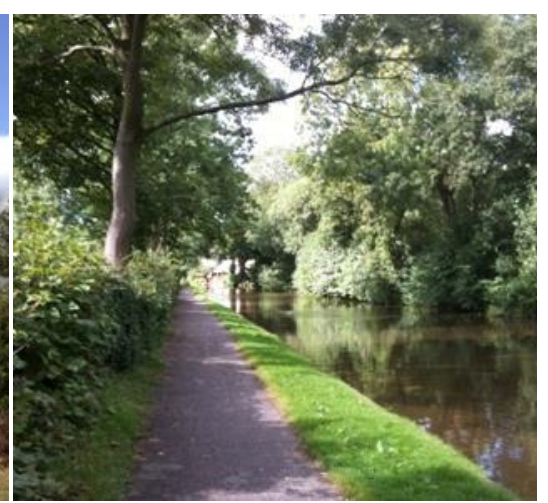

Blue

6 Figure 1. Example images of exposure environments (urban; green; blue)

7

8 


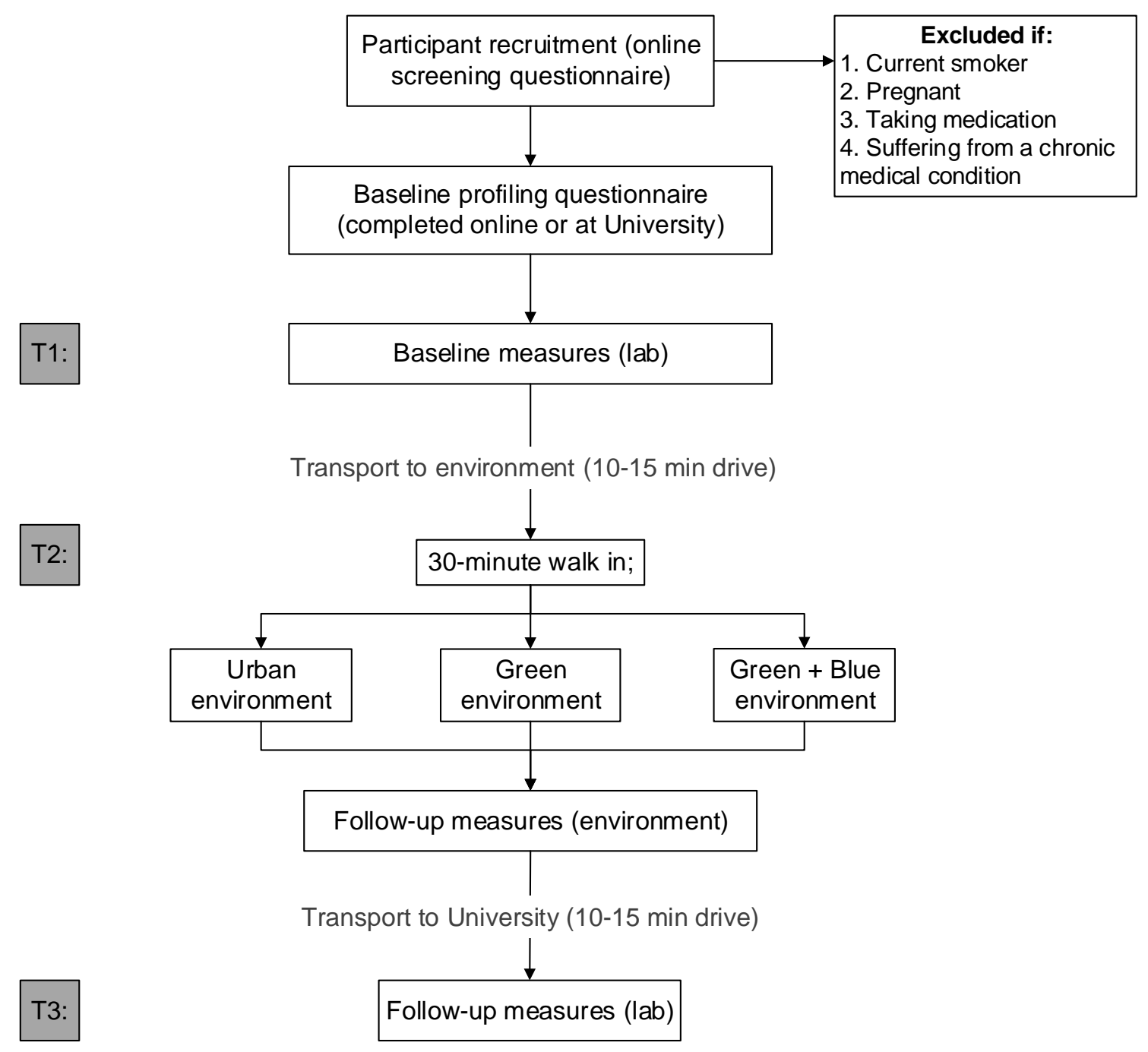

T1 (0 mins):

Baseline measures: affect (BRUMS); cognitive function (backwards digit span task); and cortisol. Ambulatory HRV monitoring begins (end of T1).

30 min walk:

CalFit monitoring starts. RPE collected at 5-minute intervals throughout exposure and social interaction monitored.

T2 (30 mins):

Follow-up measures:affect; cognitive function; and cortisol. CalFit monitoring ends (start of T2).

T3 (60 mins):

Follow-up measures (lab): affect; cognitive function; and cortisol. HRV monitoring ends (start of T3).

11 Figure 2. Flow diagram for each day of data collection 


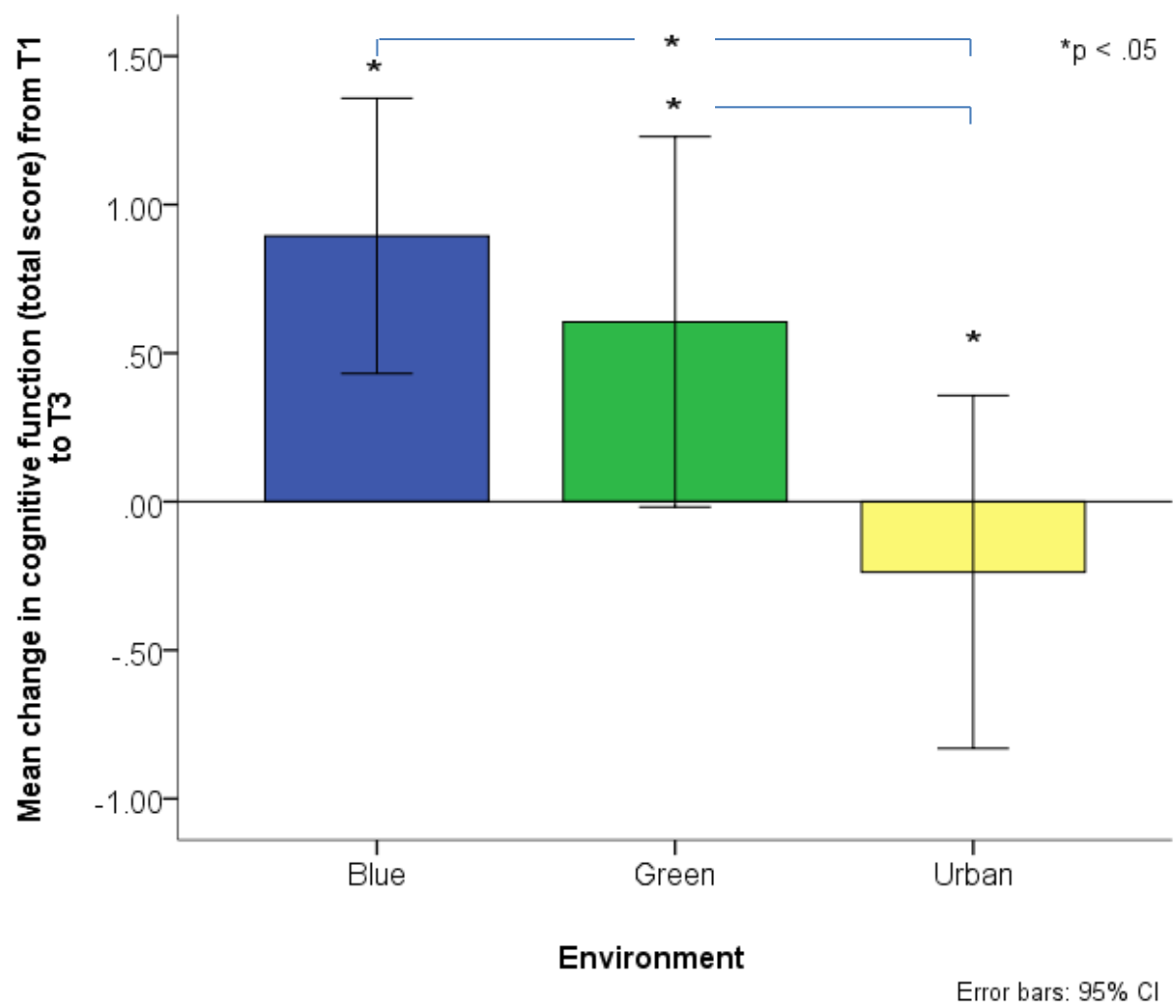

17 Figure 3. Mean change in cognitive function (backwards digit span task) from baseline to 6018 minute post exposure follow-up by environment 


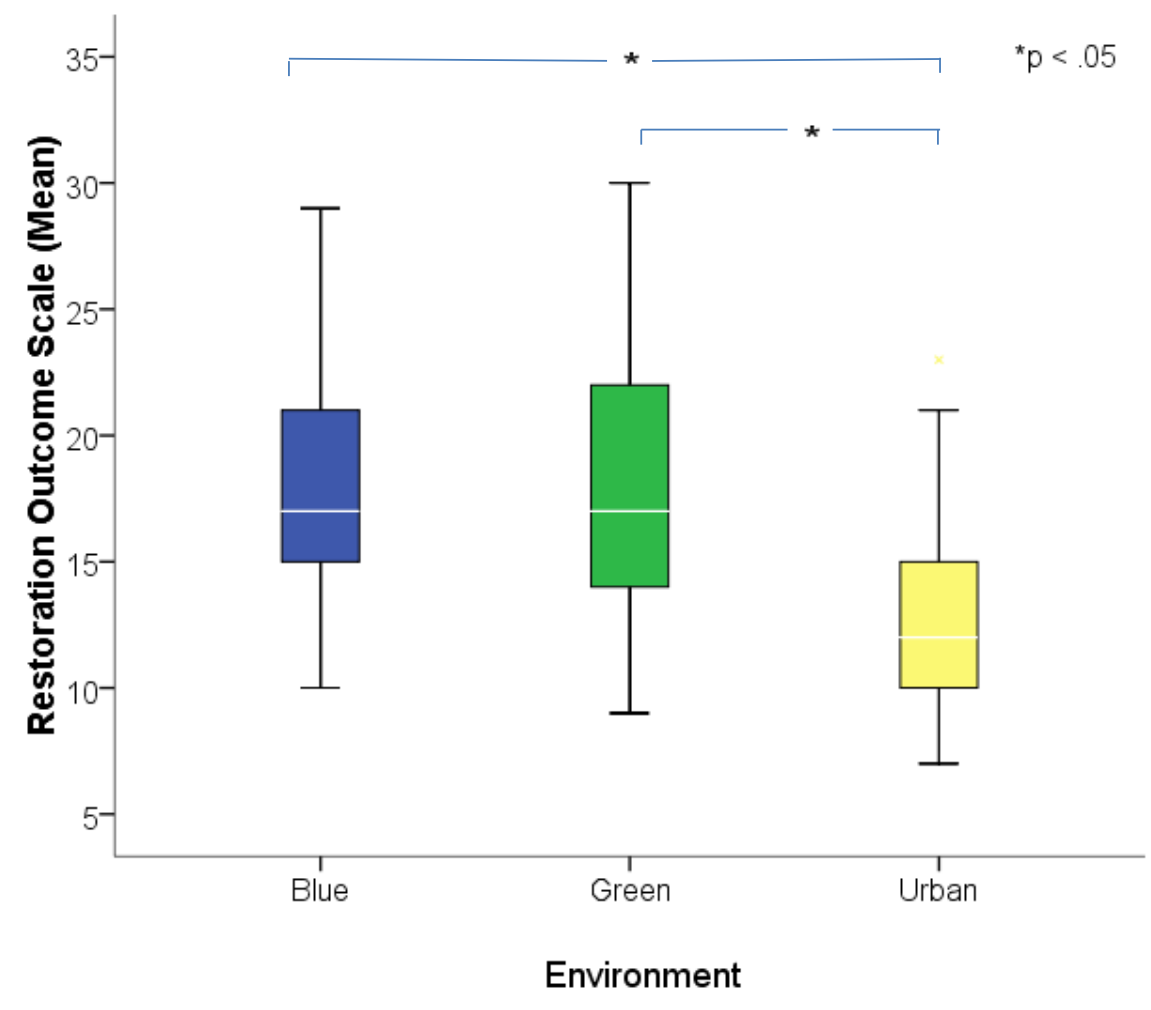

26 Figure 4. Mean restorative experience, as measured by the ROS, by environment 
Table 1. Mean (SD) values for psychological and physiological variables from baseline (T1) to 30- (T2) and 60-minute (T3) post-exposure

30 follow-up by environment

\begin{tabular}{ccccccccc}
\hline & & Cognitive & Mood & Cortisol & \multicolumn{4}{c}{ Heart Rate Variability } \\
& & Function & (TMD) & (nmol/I) & \% CCV & HFCCV & LFCCV & LFCCV:HFCCV \\
\hline \multirow{4}{*}{ Blue } & N & 38 & 35 & 37 & 34 & 34 & 34 & 34 \\
& T1 & $5.82(2.68)$ & $-4.43(4.90)$ & $5.86(3.59)$ & $7.05(3.50)$ & $0.03(0.02)$ & $0.04(0.03)$ & $1.45(1.05)$ \\
& T2 & $6.53(2.48)$ & $-6.54(4.18)$ & $3.69(1.63)$ & - & - & - & - \\
& T3 & $6.71(2.54)$ & $-5.63(3.77)$ & $3.37(1.55)$ & $7.03(3.66)$ & $0.02(0.01)$ & $0.04(0.20)$ & $1.99(0.96)$ \\
\hline \multirow{3}{*}{ Green } & T1 & $6.21(2.93)$ & $-3.00(6.31)$ & $5.71(3.14)$ & $6.45(3.29)$ & $0.03(0.02)$ & $0.03(0.02)$ & $1.79(1.28)$ \\
& T2 & $6.37(2.57)$ & $-5.34(4.03)$ & $3.87(2.17)$ & - & - & - & - \\
& T3 & $6.82(2.59)$ & $-5.26(3.23)$ & $3.53(1.88)$ & $6.67(4.07)$ & $0.02(0.02)$ & $0.04(0.03)$ & $2.17(1.02)$ \\
\hline \multirow{3}{*}{ Urban } & T1 & $6.68(5.79)$ & $-4.00(5.43)$ & $5.21(2.36)$ & $6.89(3.07)$ & $0.03(0.01)$ & $0.04(0.02)$ & $2.08(1.39)$ \\
& T2 & $6.84(2.52)$ & $-5.57(4.10)$ & $3.77(1.79)$ & - & - & - & -
\end{tabular}
high frequency powers; LFCCV, percentage coefficient of component variance for low frequency power; LFCCV:HFCCV, ratio between LFCCV and HFCCV 